\title{
Histórico da realidade virtual e seu uso em medicina
}

\author{
History of virtual reality and its use in medicine
}

José Weber V. De Faria ${ }^{1}$, Eberval Gadelha Figueiredo², Manoel Jacobsen Teixeira ${ }^{3}$

De Faria JWV, Figueiredo EG, Teixeira MJ. Histórico da realidade virtual e seu uso em medicina / History of virtual reality and its use in medicine. Rev Med (São Paulo). 2014 jul.-set.;93(3):106-14.

RESUMO: A Realidade Virtual pode ser definida como uma avançada interface homem-máquina que simula um ambiente realístico e permite que participantes interajam com ele. Essa ciência tem suas origens nas academias militares e na indústria do entretenimento, com grande impulso na segunda metade do século XX. Através de técnicas como a estereoscopia, é possível usar os sentidos humanos e emular experiências com grande fidelidade. Modelos de Realidade Virtual ganham importância cada vez maior para a medicina. Hoje é possível encontrar diversas aplicações nessa área, como planejamento pré-operatório, assistência, treinamento cirúrgico e ensino. Para o estudo da neuroanatomia e da neurocirurgia especificamente, embora os custos proibitivos e a experiência visual limitada ainda sejam realidade, a tecnologia da Realidade Virtual tem demonstrado grande eficiência e utilidade, assumindo definitivamente seu papel no contexto da educação médica.

DESCRITORES: Informática médica; Multimídia, Tecnologia; Neurocirurgia; Ensino.

\begin{abstract}
Virtual Reality can be defined as an advanced interface between man and machine, that simulates a realistic environment and allows participants to interact with such ambience. This Science has its origins in the military academies and in the entertainment industry, and experienced great impulse in the second half of twentieth century. Using techniques like stereoscopy, it is possible to use human senses and to emulate experiences with much fidelity. Models of Virtual Reality became more and more important for medicine. Today, we can find many applications in this field, such as preoperative planning, assistance, surgical training and teaching. For the study of neuroanatomy and neurosurgery in particular, the technology of Virtual Reality has shown great effectiveness and utility, despite of high costs and low quality of visual information. Virtual Reality is now definitively included in the context of medical education.
\end{abstract}

KEY WORDS: Medical informatics; Multimedia; Technology; Neurosurgery; Teaching.

\footnotetext{
${ }^{1}$ Professor Doutor da Disciplina de Neurocirurgia, Universidade Federal de Uberlândia - Uberlândia, MG.

${ }^{2}$ Professor Associado da Disciplina de Neurocirurgia, Faculdade de Medicina da Universidade de São Paulo - São Paulo, SP.

${ }^{3}$ Professor Titular da Disciplina de Neurocirurgia, Faculdade de Medicina da Universidade de São Paulo - São Paulo, SP.

Endereço para correspondência: Eberval Gadelha Figueiredo. Divisão de Clínica Neurocirúrgica - Hospital das Clínicas - FMUSP. R. Enéas Carvalho de Aguiar, 255 - 5º Andar. São Paulo, SP, Brasil. E-mail: ebgadelha@yahoo.com.
} 


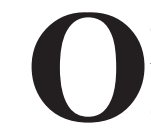
desenvolvimento tecnológico da realidade virtual teve seu início na década de 1950 e tem crescido consideravelmente nos últimos anos. Inicialmente direcionada ao entretenimento e às necessidades das forças armadas americanas, a realidade virtual hoje é utilizada em diversas aplicações médicas como planejamento pré-operatório, assistência, treinamento cirúrgico e ensino. Um dos grandes desafios para o futuro é oferecer sistemas que reproduzam exatamente aquilo que o médico vê e sente na realidade não virtual e que permita simulação em tempo real.

A realidade virtual iniciou-se com os simuladores de voo construídos pela Força Aérea dos Estados Unidos após a Segunda Guerra Mundial e na indústria de entretenimento por meio do Sensorama (Figura 1), patenteado em 1962 por Morton Heilig, um especialista em multimídia que, combinando estímulos sonoros, mecânicos e olfatórios, permitia a simulação de uma experiência multissensorial com visão estereoscópica: o usuário podia fazer um passeio pré-gravado de motocicleta por Manhattan ${ }^{1,2,3}$.

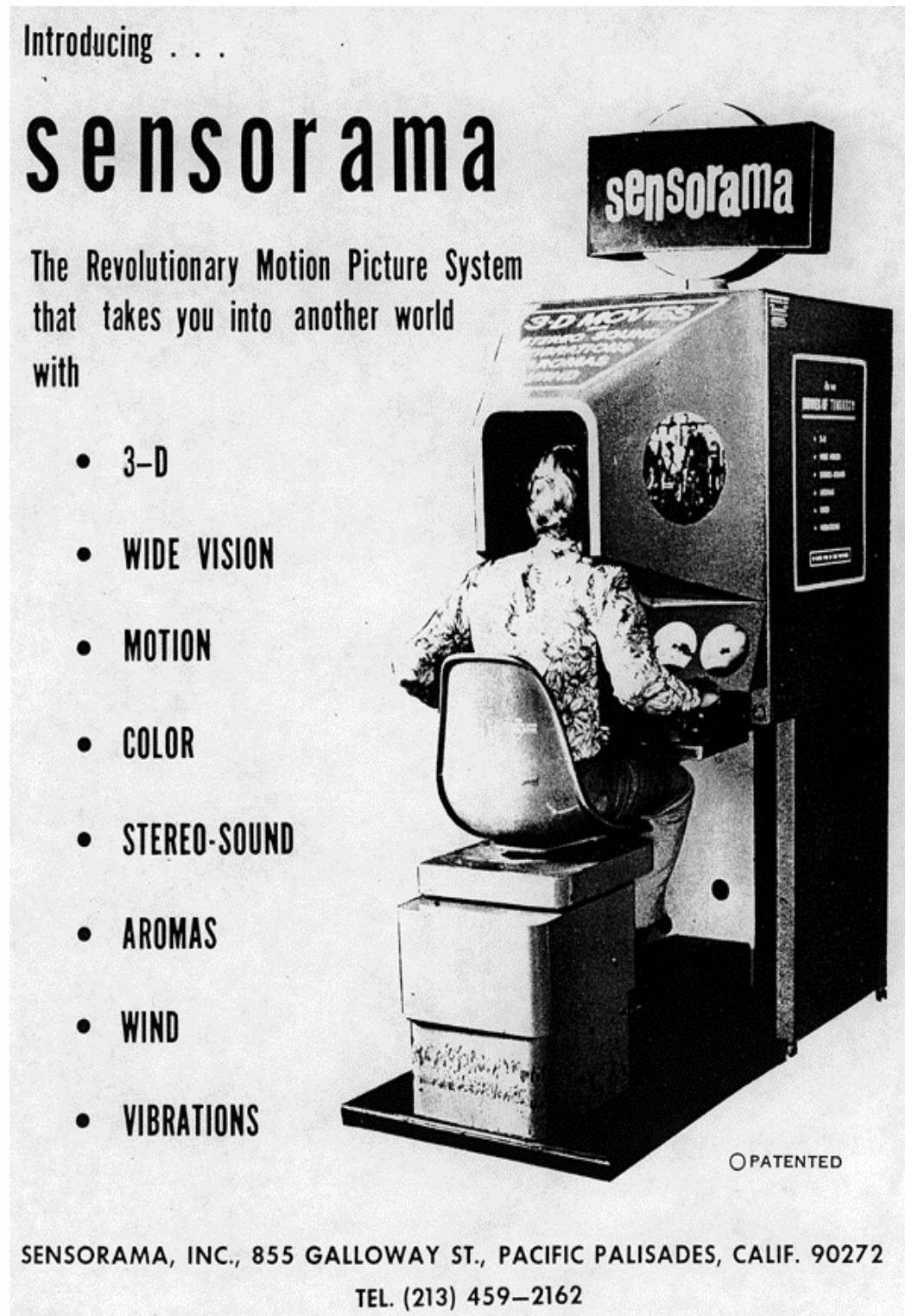

Figura 1 - Protótipo comercial do Sensorama (Fonte: Pimentel; Teixeira, 1995³)

$\mathrm{Na}$ Universidade de Utah, em meados de 1965, Ivan Sutherland apresentou sua tese de doutorado em que desenvolveu uma caneta óptica para desenhar diretamente na tela de um computador dando o primeiro passo para a criação da computação gráfica interativa e para a criação da realidade virtual ${ }^{2}$. O mesmo autor desenvolveu o primeiro 
vídeo-capacete funcional para gráficos de computador, o qual permitia ao usuário ver, através da movimentação de sua cabeça, os diferentes lados de uma estrutura de arame na forma de um cubo flutuando no espaço².

Nessa época, Myron Krueger, na Universidade de Wisconsin, iniciou estudos com realidade artificial e, em 1975, desenvolveu o Videoplace, onde uma câmera de vídeo era utilizada para filmar os participantes. A imagem bidimensional era projetada em uma grande tela. A interação dos participantes entre si e com os objetos projetados ficou conhecida por Realidade Virtual de Projeção ${ }^{1,2}$.

Com o intuito de treinar pilotos para voar e lutar, Thomas Furness em 1982 desenvolveu, para a Força Aérea Americana, o Visually Coupled Airborne Systems Simulator - VCASS, conhecido como "Super Cockpit". Trata-se de um simulador da cabine de um avião que, por meio do uso de computadores e videocapacetes interligados com áudio e vídeo, dotado de alta qualidade de resolução nas imagens e rapidez no trabalho com essas imagens, permitia o aprendizado do piloto com grande liberdade para movimentação $0^{2,4}$.

Em 1984, Michael McGreevy, utilizando-se de uma nova tecnologia de visores de cristal líquido (LCD), desenvolveu o projeto Virtual Visual Environment Display (VIVED), na NASA ${ }^{5}$. A parte de áudio e vídeo foi montada sobre uma máscara de mergulho utilizando dois visores de cristal líquido com visualização de imagens estereoscópicas e com pequenos autofalantes $\operatorname{acoplados}^{2,3}$. No ano seguinte, Scott Fisher colabora com esse projeto ao enriquecê-lo com a inclusão de luvas de dados, reconhecimento de voz, síntese de som estéreo e retorno háptico ${ }^{6}$.

Thomas Zimmerman e Scott Fisher, em 1985, desenvolveram uma luva de dados denominada Data Glove, capaz de captar a movimentação e inclinação dos dedos da mão ${ }^{1,5}$. No final de 1986 , a NASA já possuía um ambiente virtual que permitia interatividade por meio de comandos pela voz, escutar fala sintetizada, ouvir som 3D e manipular objetos virtuais diretamente por meio do movimento das mãos, ou seja, visualização pelo capacete e interatividade com a utilização das luvas ${ }^{3}$.

Após essa fase de intensa contribuição das forças aéreas americanas e da NASA no desenvolvimento inicial da realidade virtual, objetivando o treinamento de soldados, o desenvolvimento de sistemas de realidade virtual tomou rumos em direção ao comércio e pesquisas em diferentes empresas governamentais e privadas, fato ocorrido no mundo inteiro ${ }^{3}$. Organizações, de firmas de software até grandes corporações de informática, começaram a desenvolver e vender produtos e serviços ligados à realidade virtual ${ }^{1}$. Em 1989, a AutoDesk apresentava o primeiro sistema de realidade virtual baseado num computador pessoal ${ }^{1}$.

Dessa forma, a realidade virtual consolidou-se na década de 90 e, no Brasil, tem sido aplicada em exploração de petróleo, indústria aeronáutica, automobilística, por universidades e centros de pesquisa. Foi constituída uma comissão para organização da área acadêmica ligada à realidade virtual com o intuito de alavancar o desenvolvimento da realidade virtual no Brasil: Comissão Especial de Realidade Virtual-CERV. O Conselho Nacional de Desenvolvimento Científico e Tecnológico (CNPq) apoiou simpósios em realidade virtual e a uma revisão da evolução da realidade virtual no Brasil foi publicada e consta no registro dos principais acontecimentos da realidade virtual do país até o ano de $2008^{6}$.

\section{O conceito de realidade virtual}

É creditado a Jaron Lanier, músico e cientista da computação, ser o primeiro a utilizar, nos anos 80 , o termo realidade virtual com o intuito de distinguir as simulações tradicionais da computação dos mundos digitais que ele tentava criar $^{5}$. Pimentel e Teixeira ${ }^{3}$ definem realidade virtual como o uso da alta tecnologia para convencer o usuário de que ele está em outra realidade - um novo meio de "estar" e "tocar" em informações: "Virtual Reality is the place where humans and computers make contact". Latta e Oberg $^{7}$ citam Realidade Virtual como uma avançada interface homem-máquina que simula um ambiente realístico e permite que participantes interajam com ele: "Virtual Reality involves the creation and experience of environments".

Em geral, o termo Realidade Virtual refere-se a uma experiência imersiva e interativa baseada em imagens gráficas $3 \mathrm{D}$ geradas em tempo real por computador ${ }^{3}$. O principal objetivo dessa nova tecnologia é fazer com que o participante desfrute de uma sensação de presença no mundo virtual ${ }^{1}$. Para propiciar essa sensação de presença, o sistema de realidade virtual integra sofisticados dispositivos. Esses dispositivos podem ser luvas de dados, óculos, capacetes, entre outros ${ }^{2}$.

Segundo Machado et al. ${ }^{8}$, realidade virtual é uma área de pesquisa que reúne conhecimentos de várias áreas como eletrônica, computação, robótica, física, psicologia, dentre outras.

\section{Formas de realidade virtual}

Duas características utilizadas para diferenciar os sistemas de realidade virtual são a capacidade de imersão e de interatividade. A imersão pelo seu poder de prender a atenção do usuário, e a interatividade no que diz respeito à comunicação usuário-sistema ${ }^{3}$. Pimentel e Teixeira, considerando nível de imersão e de interatividade do usuário, classificam as formas de realidade virtual como: realidade virtual de Simulação (permite que o usuário se sinta no mundo virtual), de Projeção (o usuário está fora do mundo virtual, mas pode comunicar-se com 
esse), Augmented Reality (Realidade Realçada, em que há combinação de imagens do mundo real com o virtual), Telepresença (por exemplo o uso da endoscopia em pacientes), Visually Coupled Displays ("Displays Visualmente Casados") e realidade de Mesa. Pimentel e Teixeira ${ }^{3}$ vêem a realidade virtual de Mesa como um subconjunto dos sistemas tradicionais. Em vez do uso de head-mounted displays (HMDs), são utilizados grandes monitores ou algum sistema de projeção para apresentação do mundo virtual. Alguns sistemas permitem ao usuário ver imagens 3D no monitor através do uso de óculos lightweight (baixo peso) ou obturadores de cristal líquido $(\mathrm{LCD})^{2}$.

\section{Estereoscopia}

Estereoscopia é a ciência (e arte) que trabalha com imagens para produzir um modelo visual tridimensional com características análogas às características da mesma imagem quando vista através da visão binocular real ${ }^{2}$. O ser humano visualiza o ambiente e os objetos ao seu entorno em profundidade ou em três dimensões. Isso é possível porque somos dotados de uma visão binocular, que surgiu no processo evolutivo quando os olhos dos animais se posicionaram na frente da cabeça. Os olhos do ser humano estão separados em média por uma distância de $64 \mathrm{~mm}$, por isso cada olho enxerga o mundo de uma maneira ligeiramente diferente um do outro. O sistema de visão binocular nos permite calcular com boa exatidão a que distância um objeto se encontra de nós e se múltiplos objetos estão mais próximos ou mais distantes. As ondas de luz penetram no olho e alcançam os fotorreceptores na retina, ativando células que enviam informações ao cérebro, as quais ativarão células que permitem a percepção da forma, cor, profundidade e movimento do objeto. Isso ocorre em áreas denominadas de córtex visual secundário e córtex de associação, nos lobos temporal e parietal. O cérebro, dessa forma, ajusta essas duas imagens, fazendo com que o ser humano seja capaz de perceber o mundo em profundidade ${ }^{1,9}$.

Nos últimos anos, as tecnologias digitais evoluíram ao ponto de imitar a visão humana estereoscópica. Atualmente pode-se obter o efeito da estereoscopia através de dispositivo e técnicas artificiais. Essas tecnologias têm o intuito de tornar a imagem mais realista por meio da obtenção do senso de profundidade. As imagens $3 \mathrm{D}$ computadorizadas estereoscópicas dão a noção de profundidade e parecem flutuar no espaço diante da superfície na qual são apresentadas ${ }^{1}$. Nas imagens estereoscópicas, a quantidade de paralaxe, distância entre imagens esquerda e direita, determina a distância aparente dos objetos virtuais em relação ao observador. $\mathrm{O}$ cérebro reúne as duas imagens em uma, sendo que essa parece ter características de profundidade, distância, posição e tamanho. Uma paralaxe menor, por exemplo, resulta na ilusão de que o objeto está distante1.

$\mathrm{O}$ primeiro passo na criação de uma imagem estereoscópica é a construção de um par de imagens, isto é, as imagens direita e esquerda do mesmo objeto são captadas em diferentes ângulos de visão. Utilizase, tradicionalmente, de uma câmera fotográfica que é deslocada ao longo de uma barra deslizante ou trilho, por meio de um eixo paralelo, obtendo-se uma primeira imagem no ponto inicial e a última no ponto final ${ }^{9,10}$.

Esse procedimento é análogo à captação da imagem pela pupila direita e esquerda. A distância de deslocamento da câmera na barra relaciona-se à distância em que o objeto se encontra da câmera, de tal sorte que, quanto mais distante o objeto do usuário maior o deslocamento necessário para a visualização estereoscópica e, quanto mais próximo o objeto da câmera, menor a necessidade de deslocamento. Outra forma é denominada captação em eixo convergente, em que o autor desloca a câmera no eixo paralelo sobre o trilho, roda a câmera em torno de $30^{\circ}$ de convergência, focando um ponto previamente determinado. Na configuração de câmeras convergentes, ocorre um efeito indesejado devido ao deslocamento vertical (paralaxe vertical) dos pontos das imagens esquerda e direita, sendo uma fonte de desconforto para o observador ${ }^{10}$.

Quanto à distância de deslocamento da câmera no trilho, existe uma regra matemática que orienta deslocar a máquina fotográfica, para direita e esquerda, por uma distância igual a 1/30 da distância entre a câmera e o objeto, embora os recursos dos softwares mais modernos permitam deslocar essas imagens no sentido de aproximálas ou afastá-las, melhorando a qualidade da visão ${ }^{8,9,11}$.

Para a obtenção do estereopar com o microscópio cirúrgico, há serviços que utilizam o deslocamento do microscópio para fazer a varredura da área a ser visualizada, com as câmeras fotográficas adaptadas aos canais de visualização do microscópio, alinhadas conforme um ponto de referência no espécime, prevenindo distorções ou piora na qualidade do par estereoscópico (estereopar) ${ }^{12-15}$. Outra forma de aquisição de estereopares é com a utilização de máquinas fabricadas para fotografias tridimensionais, as quais já disponibilizam duas objetivas, com uma distância entre elas próxima da distância interpupilar.

Em realidade virtual, a visão estereoscópica é um importante fator na determinação do nível de imersão do sistema ${ }^{16}$. No entanto, deve-se levar em conta que, na maioria dos sistemas, exibir imagem separada para olho esquerdo e direito exige do hardware o dobro de potência de processamento de imagem ${ }^{1}$. Há vários softwares disponíveis no mercado e de fácil acesso via internet que permitem a construção de estereopares e seu armazenamento para utilização em uma de suas diversas modalidades. Um editor e visor de imagens estereoscópicas versátil é o Stereo Photo Maker que pode funcionar em sistemas operacionais Windows e Mac OS. Ele permite 
ao usuário criar paginas na internet e seu download é gratuito. Realizamos um levantamento dos endereços de home page referentes a softwares que trabalham com imagens estereoscópicas (Tabela 1).

Tabela 1 - Alguns softwares de estereoscopia disponíveis na web e os endereços para download

\begin{tabular}{ll}
\hline Software & Endereço \\
\hline Fractint & nahee.com/spanky/www/fractint \\
\hline $\begin{array}{l}\text { Wiggle Stereoscopic } \\
\text { Viewer }\end{array}$ & wiggle.sourceforge.net \\
\hline AnaBuilder & anabuilder.free.fr \\
\hline Stereomerger & stereomerger.com \\
\hline $\begin{array}{l}\text { The Stereoscope } \\
\text { Applet }\end{array}$ & stereofoto.de/sapplet \\
\hline $\begin{array}{l}\text { Plascolin } \\
\text { Split MPO }\end{array}$ & stereoscopy.com/downloads \\
\hline $\begin{array}{l}\text { Callipigian 3D Photo } \\
\text { Editing }\end{array}$ & stereoscopy.com/downloads \\
\hline $\begin{array}{l}\text { Woolly Anaglyph } \\
\text { Maker }\end{array}$ & somerset3d.co.uk/wam \\
\hline \begin{tabular}{l} 
Z-Anaglyph \\
rosset.org/graphix/anaglyph/ \\
\hline
\end{tabular}
\end{tabular}

A visão estereoscópica pode ser obtida de forma indireta de cinco maneiras diferentes: estereoscopia voluntária, por anáglifo, por polarização da luz, por luz intermitente e por holografia. Nos quatro primeiros processos, cada um dos olhos do observador verá uma imagem diferente de um mesmo objeto e seu cérebro as fundirá em uma única imagem 3D. No processo por holografia a cena em 3D é registrada numa única imagem, não havendo a necessidade de um par estereoscópico, e por esta razão considerada, por alguns autores, uma forma não estereoscópica de apresentação da imagem tridimensional $^{17}$.

Anáglifo é o nome dado a figuras planas cujo relevo se obtém por cores complementares, normalmente vermelho e verde ou vermelho e azul esverdeado. Nesse caso, cada um dos olhos utilizará um filtro diferente para visualizar as imagens do par estereoscópico: o olho que estiver com o filtro vermelho refletirá apenas a cor vermelha e o olho que estiver com o filtro verde/azul refletirá apenas a imagem verde/azul. Assim, as duas imagens são separadas na observação e fundidas em uma única imagem 3D preto e branco ${ }^{10,18}$. As projeções com o método anaglífico são feitas a partir de uma única imagem constituída pela fusão das imagens direita e esquerda em telas comuns, requerendo apenas o uso de óculos com os filtros coloridos, e essa imagem tem como principais limitações a reprodução de cores já mencionada e a consequente menor luminosidade, fatos que comprometem a qualidade final da imagem, mas não limitam a produção do efeito 3D estereoscópico (Figura 2) ${ }^{19}$.

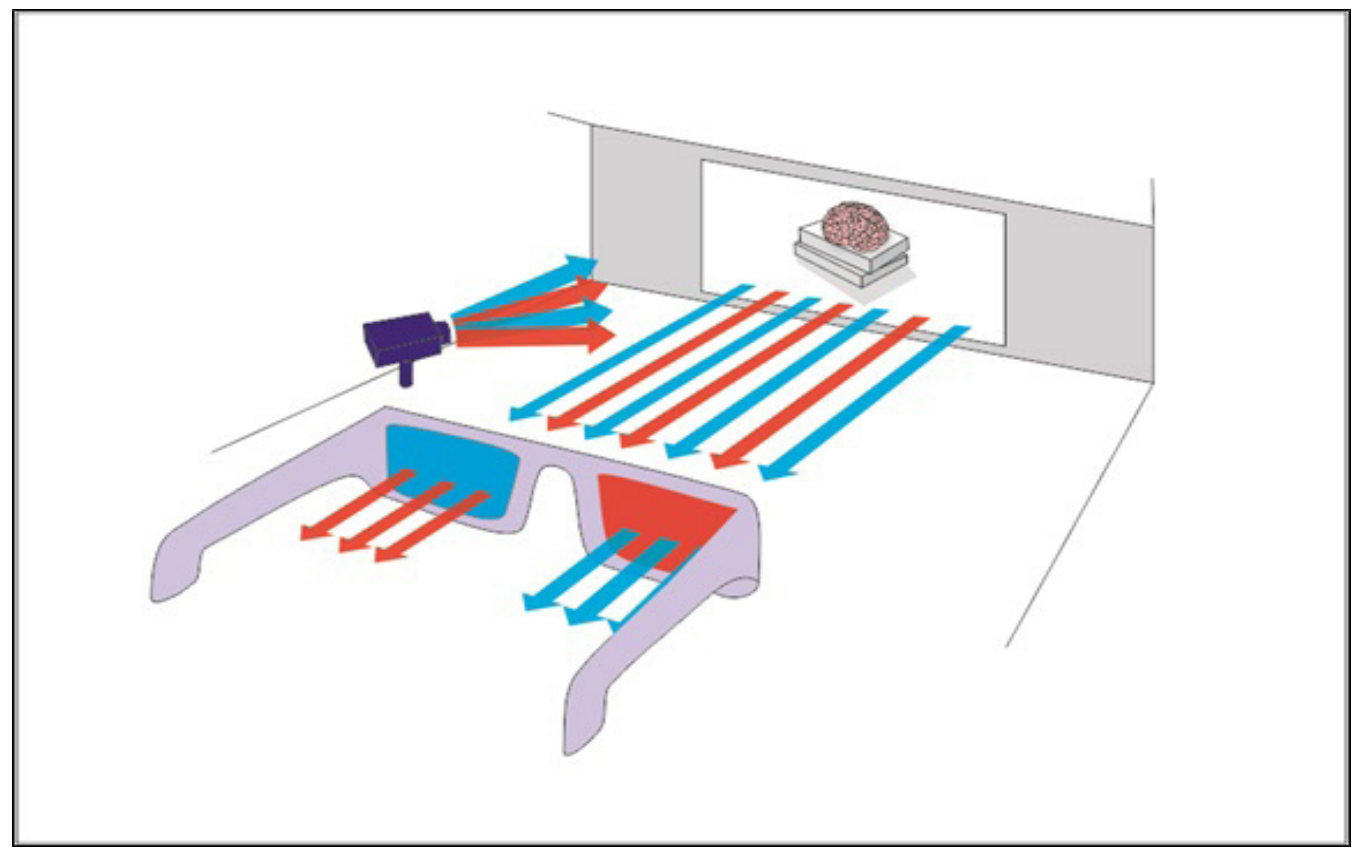

FIGURA 2 - Projeção e visualização de uma imagem estereoscópica em anáglifo vermelho/azul. Lentes azul e vermelha filtram as duas imagens projetadas, permitindo que apenas uma delas alcance cada olho 
No processo de estereoscopia por luz polarizada, são utilizados filtros polarizadores que fazem com que a projeção das imagens do par estereoscópico sejam polarizadas em planos ortogonais (por exemplo um plano vertical e um horizontal). Dessa forma, o observador utiliza filtros polaróides ortogonais correspondentes aos planos de projeção e vê com cada olho apenas uma das imagens projetadas. Da fusão das imagens vistas por cada olho resultará a visão estereoscópica. Na prática as imagens armazenadas em um computador são transferidas por uma placa de vídeo dual, que permite exibir imagens para dois monitores ao mesmo tempo, através de duas saídas de vídeo até dois projetores. Aos dois projetores conectamse dois filtros polarizadores que irão direcionar a luz à tela que não cause a sua despolarização, geralmente telas metalizadas, que irão refletir a luz polarizada aos óculos com lentes polarizadas, fazendo com que cada olho veja apenas um sentido de polarização (Figura 3$)^{17}$.

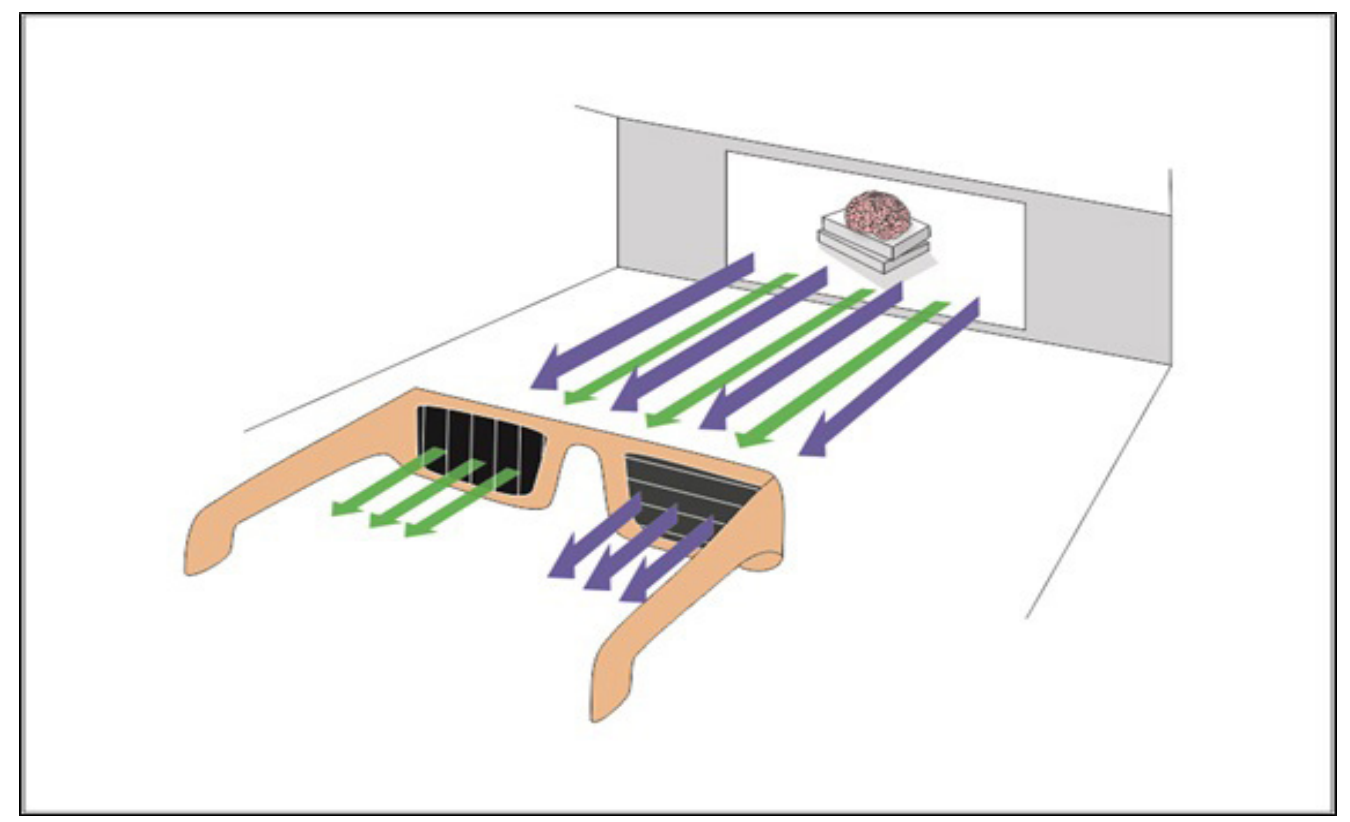

Figura 3 - Os óculos polarizados permitem apenas uma das imagens em cada olho, porque cada lente tem uma polarização diferente

No processo de Estereoscopia por Luz Intermitente, ou campos sequenciais alternados (field sequential technique), as imagens são projetadas alternadamente para cada olho a um intervalo de $1 / 20$ a $1 / 60$ segundos, de modo que cada olho possa ver apenas uma imagem. Assim, o observador não perceberá a alternância das imagens e verá uma única imagem 3D. Uma grande vantagem desse processo sobre o uso de anáglifos é a qualidade das imagens coloridas. Para a visualização estereoscópica em campos sequenciais faz-se necessário um óculos denominado de cristal líquido, que funciona como um obturador, abrindo e fechando conforme o estereograma direito ou esquerdo enviado. O controle para a sincronia entre o gerador da imagem (ex.: tela de uma TV digital 3D) e os óculos é feito comumente através de sinalização de raios infravermelho ${ }^{17-19}$.

A holografia, inventada em 1947 pelo físico Denis Gabor, não se utiliza de um par de imagens estereoscópicas, uma vez que a holografia é uma técnica que registra em filme a informação relativa a um objeto ou cena; diferente da estereoscopia, que é a cópia da realidade através da fotografia, a holografia representa a realidade através de uma criação do ambiente visível de qualquer perspectiva de observação, não se restringindo àquele visualizado no espaço real capturado pelos outros sistemas. A holografia capta as informações de uma imagem tridimensional, incluindo profundidade, e as grava também em $3 \mathrm{D}^{17}$.

\section{Criação da realidade virtual}

Para a criação de um mundo virtual interativo, é necessário criar um ambiente e habilitá-lo com objetos e características virtuais ${ }^{20}$. O aplicativo de realidade virtual é uma simulação animada que permite definir e exibir um objeto 3D, alterar seu ponto de referência e campo de visão, manipular e interagir com os objetos e fazer com que esses objetos afetem uns aos outros ${ }^{1}$. O software de realidade virtual permite permear objetos com comportamentos (propriedades físicas) e programá-los para ativar algum tipo de feedback visual, auditivo ou tátil quando um evento específico acontece, além de gerenciar toda a sequência de eventos. A maioria dos sistemas de construção de mundos virtuais compartilha alguns conceitos básicos que caracterizam o desenvolvimento da realidade virtual 
e que permitem aos desenvolvedores a criação de uma simulação bastante realística ${ }^{2}$. Segundo Jacobson ${ }^{1}$, esses conceitos básicos poderiam ser: o universo e seus objetos, técnicas de apresentação e dinâmicas e feedback. Além de compartilharem os conceitos acima citados, os softwares para criação de realidade virtual também costumam oferecer recursos para determinados tipos de dispositivos de interação, permitindo programá-los para ativar algum tipo de feedback visual, auditivo ou tátil ${ }^{1}$.

\section{Aplicações da realidade virtual na neuroanatomia e na neurocirurgia}

Especificamente na neurocirurgia, vários sistemas de simulação cirúrgica têm sido desenvolvidos. Apesar de úteis, o custo desses sistemas são proibitivos, sua disponibilização é restrita e a experiência visual que eles oferecem é limitada. Para a educação em neurocirurgia, têm sido desenvolvidos novos instrumentos que permitem demonstração da complexa anatomia 3D e suas relações, buscando, em associação com a estereoscopia recriar a experiência de acessos cirúrgicos de forma mais realística do que os métodos de ensino tradicionais. Balogh e colaboradores $^{21,22}$ e Henn et al..$^{23}$, têm sido pioneiros nos trabalhos envolvendo anatomia microcirúrgica com aquisição de imagens do sítio cirúrgico e de peças anatômicas através do uso de um microscópio robótico. Porém, o custo desse método é alto, envolve uma equipe de engenheiros, laboratório sofisticado e realidade gráfica para renderização de imagens.

Com o objetivo de reduzir os riscos nas neurocirurgias e melhorar a acurácia do procedimento, Zamorano et al. ${ }^{24}$ em 1994 criaram um software que permite o planejamento cirúrgico com a utilização de um arco estereotático ou através da utilização de mão livre guiada por infravermelho. Esse software propicia o mapeamento das informações dos estudos de imagem e forma uma nova imagem tridimensional, possibilitando acompanhar a trajetória planejada com a posição do instrumento em tempo real no monitor do computador ${ }^{24}$.

Kockro et al. ${ }^{25}$ em 2000 publicaram um estudo com planejamento pré-operatório e simulação de cirurgias com realidade virtual. Os autores ${ }^{25}$, utilizando um sistema de planejamento neurocirúrgico desenvolvido pela Dextroscope ${ }^{\circledR}$, que permite a fusão de múltiplas imagens de tomografia computadorizada (TC) e ressonância nuclear magnética (RNM) e a sua manipulação em tempo real, em uma interface denominada Visual Intracranial Visualization and Navegation (VIVIAN), que produz uma terceira imagem 3D e estereoscópica, conseguiram simular visões intraoperatórias da cirurgia, úteis para o entendimento das relações da lesão com as estruturas vizinhas e a melhor porta de entrada para o acesso cirúrgico.

Baseados no conceito de que a realidade virtual oferece um grande potencial para o treinamento cirúrgico, Li et al. $^{26}$, em 2002, desenvolveram um sistema para treinamento de neurocirurgiões na realização de rizotomia percutânea do nervo trigêmeo. O treinamento envolveu a inserção de uma agulha através do forame oval e a lesão do nervo. O servidor ofereceu ao usuário o retorno de seu desempenho na realização do procedimento ${ }^{26}$.

Com o objetivo de conhecer os melhores pontos de referência externa para direcionamento de uma agulha de punção ventricular durante ventriculostomias posteriores, Lee et al. ${ }^{27}$ criaram um modelo que, a partir de reconstrução de imagens de RNM e de um sistema de planejamento estereoscópico 3D, marca o ponto de entrada e permite a realização de várias trajetórias simuladas, permitindo a análise da melhor trajetória para alcançar o átrio ou o corpo do ventrículo lateral. Concluíram que, entrando pelo ponto de Frazier, a melhor trajetória ocorre quando o alvo está a 4 centímetros do canto medial contralateral; quando a entrada é o ponto de Dandy e o melhor alvo está a 2 centímetros acima da glabela ${ }^{27}$.

Com o objetivo de estudar a utilidade da realidade virtual em procedimentos da base do crânio, Rosahl et al. ${ }^{28}$, em 2006, publicaram um estudo em que o procedimento foi guiado por imagens volumétricas criadas a partir de tomografias, ressonâncias e angiografias dos próprios pacientes, que apresentavam lesões da fossa anterior, posterior e média. As imagens foram trabalhadas no sentido de oferecer um campo de acesso operatório virtual, que era consultado pela equipe durante a cirurgia e comparado

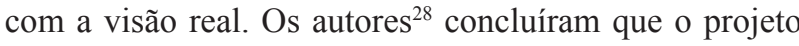
não substitui o conhecimento da neuroanatomia, mas é útil como apoio para o procedimento neurocirúrgico.

Kakizawa et al. ${ }^{29}$ em 2007 criaram um atlas 3D e interativo da base do crânio, utilizando um software comercialmente disponível para ilustração (Maya 6.0). Os autores, a partir da dissecação do cadáver, desenvolveram um modelo de alta resolução incluindo estruturas como os nervos cranianos, núcleos do tronco, vasos sanguíneos, dentre outros. O modelo pode ser manipulado, usa transparência que permite ver estruturas mais profundas e é útil como ferramenta de ensino para estudantes e residentes para aprendizado da neuroanatomia e entendimento de acessos cirúrgicos ${ }^{29}$.

Vloeberghs et al. $^{30}$ (cientistas de computação, engenheiros mecânicos, especialistas em desenho gráfico e um neurocirurgião), em 2007, criaram um simulador de cirurgia que permite realizar alguns passos cirúrgicos com retorno háptico, utilizado como ferramenta de treinamento. Também em 2007, Sengupta et al. ${ }^{31}$ criaram um simulador de procedimentos endovasculares. Por meio de banco de dados que simula a força aplicada em procedimentos endovasculares e a utilização de material sintético, criou-se uma ferramenta para facilitar o planejamento pré-operatório, aumentar a segurança na intervenção e permitir o treinamento de novos cirurgiões. 
Para se quantificar o ganho visual com a retração encefálica em cirurgias da base do crânio, D'Ambrosio et al..$^{32}$ em 2008 simularam a craniotoma frontotemporal orbitozigomática (CFTOZ) em ambiente virtual e tridimensional. Compararam o ganho visual com a remoção óssea e com diferentes níveis de retração. Para isso, construíram um modelo gráfico do crânio e do cérebro, dos lobos frontal e temporal, incorporaram imagens de tomografia de crânio em cortes sagitais e axiais reconstruídos tridimensionalmente para visualização dos limites da craniotomia. Cada passo necessário para a craniotomia foi criado com medidas de comprimento e angulação de visão, cada retração cerebral foi também simulada, até a visualização das estruturas profundas. Concluíram que há um ganho significativo de visão com a CFTOZ e com a retração do parênquima ${ }^{32}$.

Em 2010, Malone et al. ${ }^{33}$ publicaram uma excelente revisão de ambientes de simulação baseados em computadores e sua aplicação cirúrgica. Os autores ${ }^{32}$ chamaram a atenção para o fato de que a evolução nessa área está ligada à criação de volumes a partir de computação gráfica, ao desenvolvimento de modelos de

\section{REFERÊNCIAS}

1. Jacobson L. Realidade virtual em casa. Rio de Janeiro: Berkeley; 1994.

2. Machado LS. Conceitos básicos da realidade virtual [monografia]. São José dos Campos: Instituto Nacional de Pesquisas Espaciais; 1995. (INPE-5975-PUD/025). Disponível em: http://www.di.ufpb.br/liliane/ publicacoes/1995_rt.pdf.

3. Pimentel K, Teixeira K. Virtual reality: through the new looking glass. 2a ed. New York: McGraw-Hill; 1995.

4. Kocian DF. A visually-coupled airborne systems simulator (VCASS): an approach to visual simulation. In: Air Force Aerospace Medical Research Lab Wright-Patterson AFB OH, Williams, Phoenix, Az, 18 May 1977. Conference paper. Williams, Az; 1977. Available from: http://goo.gl/ c5GAiO.

5. Fisher SS, McGreevy M, Humpries J, Robinett W. Virtual environment display system. In: ACM 1986 Workshop on Interactive 3D Graphics, Chapel Hill, North Carolina, Oct 23-24, 1986. Available from: http://itofisher.com/sfisher/ portfolio/files/images/7/Virtual_Environment_Display.pdf.

6. Kirner C. Evolução da realidade virtual no Brasil. In: Proceedings of the 10th Symposium on Virtual and Augmented Reality, 2008. Porto Alegre: SBC; 2008. v.1, p.1-11.

7. Latta JN, Oberg DJ. A conceptual virtual reality model. IEEE Comput Graph Appl. 1994;14(1):23-9. doi: deformação tecidual e à capacidade do sistema de gerar retorno háptico. Os autores ${ }^{33}$ comentaram que os custos seriam menores se talvez no futuro houvesse uma parceria internacional para a construção de uma plataforma comum para simulação. Uma das vantagens da construção da plataforma comum seria a redução de custos.

O desenvolvimento da neurocirurgia caminha com o desenvolvimento da tecnologia de ponta, o que requer um ambiente socioeconômico favorável, um planejamento de gastos visando às intenções para o futuro, tendo em vista o alto custo desta tecnologia ${ }^{34}$. A revolução digital dos últimos 20 anos nos oferece novas ferramentas intelectuais e práticas, novos desenhos, nova arquitetura, nova complexidade que permite a sofisticação e modernização da neurocirurgia ${ }^{35}$. Modelos tridimensionais para planejamento neurocirúrgico foram utilizados na escolha e treinamento de um procedimento neurocirúrgico com resultados favoráveis levando à melhor escolha, entendimento da complexidade das lesões intracranianas e a experiência com o procedimento foi favorável ao ponto do autor descrever como uma sensação de "déjà-vu"36.
$10.1109 / 38.250915$.

8. Machado LS, Campos SF, Cunha ILL, Moraes RM. Cybermed: realidade virtual para ensino médico. IFMBE Proc. 2004;5(1):573-6. Available from: http://www.di.ufpb. br/liliane/publicacoes/2004_IFMBE.pdf.

9. Reeve S, Flock J. Basic principles of stereoscopic 3D. Londres: British Sky Broadcasting; 2010. Available from: http://www.sky.com/shop/export/sites/www.sky.com/ shop/_PDF/3D/Basic_Principles_of_Stereoscopic_3D_ v1.pdf.

10. Maschio VA. A estereoscopia: investigação de processo de aquisição, edição e exibição de imagens estereoscópicas em movimento [dissertação]. Bauru: Universidade Estadual Paulista Júlio de Mesquita Filho; 2008. Disponível em: http://www.faac.unesp.br/Home/Pos-Graduacao/Design/ Dissertacoes/alexandre.pdf.

11. Figueiredo EG, Faria JW, Ballester G, Teixeira MJ. Quicktime VR TM: when medical education meets virtual reality. Rev Med (São Paulo). 2009;88(Suppl.):175-80. doi: http:// dx.doi.org/10.11606/issn.1679-9836.v88i3/4p175-180.

12. Aziz MA, McKenzie JC, Wilson JS, Cowie RJ, Ayeni SA, Dunn BK. The human cadaver in the age of biomedical informatics. Anat Rec (New Anat). 2002;269:20-32. Doi 10.1002/AR.10046.

13. Rhoton Jr AL. Cranial anatomy and surgical approaches. Rio de Janeiro: Di Livros; 2009. 
14. Winkelmann A. Anatomical dissection as a teaching method in medical school: a review of the evidence. Med Educ. 2007;41:15-22. doi: 10.1111/j.1365-2929.2006.02625.x.

15. Granger NA. Dissection laboratory is vital to medical gross anatomy education. Anat Rec (Part B: New Anat). 2004;281B:6-8. doi: 10.1002/ar.b.20039.

16. Lamperti A, Sodicoff M. Computer-based neuroanatomy laboratory for medical students. Anat Rec. 1997;249:422-8. doi: 10.1002/(SICI)1097-0185(199711)249:33.3.CO;2-H

17. Rizzolo LJ. Human dissection: an approach to interweaving the traditional and humanistic goals of medical education. Anat Rec. 2002;269:242-8. doi 10.1002/ar.10188.

18. Ribas GC, Ribas EC, Rodrigues Jr AJ. O cérebro, a visão tridimensional, e as técnicas de obtenção de imagens estereoscópicas. Rev Med. 2006;85(3):78-90. doi: http:// dx.doi.org/10.11606/issn.1679-9836.v85i3p78-90.

19. Meneses MS, Cruz AV, Castro IA, Pedrozo AA. Estereoscopia aplicada à neuroanatomia: estudo comparativo entre as técnicas de filtro de cores e de polarização. Arq Neuropsiquiatr. 2002;60(3-B):769-74. http://dx.doi. org/10.1590/S0004-282X2002000500017.

20. Ribas GC, Bento RF, Rodrigues Jr. AJ. Anaglyphic 3D stereoscopic printing: revival of an old method for anatomical and surgical teaching and report. J Neurosurg. 2001;95(6):1057-66. Available from: http://thejns.org/doi/ pdf/10.3171/jns.2001.95.6.1057.

21. Balogh AA, Preul MC, Schornak M, Hickman M, Spetzler RF. Intraoperative stereoscopic quicktime virtual reality. J Neurosurg. 2004;100(4):591-6. doi: 10.3171/ jns.2004.100.4.0591

22. Balogh AA, Preul MC, László K, Schornak M, Hickman M, Deshmukh P, et al. Multilayer image grid reconstruction technology: four-dimensional interactive image reconstruction of microsurgical neuroanatomic dissections. J Neurosurg. 2006;58(Suppl.):157-65. doi: 10.1227/01. NEU.0000193514.07866.F0.

23. Henn JS, Lemole GM, Ferreira MAT, Gonzáles LF, Schornak M, Preul MC, et al. Interactive stereoscopic virtual reality: a new tool for neurosurgical education technical note. J Neurosurg. 2002;96(1):144-9. doi: 10.3171/ jns.2002.96.1.0144.

24. Zamorano L, Jiang Z, Kadi M. Computer-assisted neurosurgery system: Wayne State University hardware and software configuration. Comput Med Imag Graph. 1994;18:257-71. doi: 10.1016/0895-6111(94)90050-7.

25. Kokro RA, Serra L, Tseng-Tsai Y, Chan C, Yih-Yian S, Gim-
Guan C, et al. Planning and simulation of neurosurgery in a virtual reality environment. Neurosurgery. 2000;46:118-35. DOI: 10.1097/00006123-200001000-00024.

26. Li Y, Brodie K, Phillips N. Web-based VR training simulator for percutaneous rhizotomy. Stud Health Technol Inform. 2002;85:261-7. doi: 10.3233/978-1-60750-914-1-175.

27. Lee CK, Tay LL, Ng WH, Ng I, Ang BT. Optimization of ventricular catheter placement via posterior approaches: a virtual reality simulation study. Surg Neurol. 2008;70:274-7. doi: 10.1016/j.surneu.2007.07.020.

28. Rosahl SK, Gharabaghi A, Hubbe U, Shahidi R, Samii M. Virtual reality augmentation in skull base surgery. Skull Base. 2006;16:59-66. doi: 10.1055/s-2006-931620.

29. Kakizawa Y, Hongo K, Rhoton Jr AL. Construction of a three-dimensional interactive model of skull base and cranial nerves. Neurosugery. 2007;60:901-10. doi: 10.1227/01. NEU.0000255422.86054.51.

30. Vloeberghs M, Glover A, Benford S, Jones A, Wang P, Becker A. Virtual neurosugery training for the future. Br J Neurosurg. 2007;21:262-7. doi:10.1080/02688690701245824.

31. Sengupta A, Kesavadast T, Hoffman K, Baier R, Schafer S. Evaluating tool-artery interaction force during endovascular neurosurgery for developing haptic engine. Studies Health Technol Inform. 2007;125: 418-20. Available from: http:// goo.gl/uJtdis.

32. D'Ambrosio AL, Mocco J, Hankinson TC, Bruce JN, Van Loveren HR. Quantification of the frontotemporal orbitozygomatic approach using a three-dimensional visualization and modeling application. Neurosurgery. 2008;62(Suppl.):251-60. doi: 10.1227/01.neu.0000317401. 38960.f6.

33. Malone HR, Syed ON, Downes MS, D'Ambrosio AL, Quest DO, Kaiser MG. Neurosurgery. Simulation in neurosurgery: a review of computer-based simulation environments and their surgical applications. 2010;67(4):1105-16. doi: 10.1227/NEU.0b013e3181ee46d0.

34. Apuzzo MLJ. New dimensions of neurosurgery in the realm of high technology: possibilities, practicalities, realities. Neurosurgery. 1996;38:625-39. Available from: http://goo. gl/TOrkT4.

35. Apuzzo MLJ. Reinventing neurosurgery: entering the third millennium. Neurosurgery. 2000;46:1-2. doi: 10.1097/00006123-200001000-00001.

36. Stadie AT, Kokro RA, Reish R, Tropine A, Boor S, Stoeder P, et al. Virtual reality system for planning minimally invasive neurosurgery [technical note]. J Neurosurg. 2008;108:38294. doi: $10.3171 / \mathrm{JNS} / 2008 / 108 / 2 / 0382$. 